\title{
The New Generation of Drug Discovery and its Analytical Technologies
}

\author{
Yibin Feng* and Ning Wang \\ LKS Faculty of Medicine, School of Chinese Medicine, The University of Hong Kong, 10 Sassoon Road, Pokfulam, Hong Kong
}

The pharmaceutical manufacturing is receiving more and more challenges today. The rising customer expectation makes the commercial environment of healthcare providers gets harsher. Increasing wants of healthcare payers on new alternatives that are clinically and economically better than the current therapies imposes the new costs constraints on the pharmaceutical companies. On the other hand, unchanged discovery and developing process in finding novel alternatives over the past decades makes the scientific productivity be expected desirable. During the past years, the pharmaceutical manufacturing's outputs remain stable and seems hardly to soar all of a sudden. As a result, the number of approved new molecules and biological licence applications by FDA is decreasing year by year. Compared with 1996, when 53 new molecular entities were approved, the number has been reduced to 19 in 2009. Pharmaceutical companies are suffering from a very low success rate in developing potential candidates that are screened from a large pool of chemical database, towards new drug products. New demands are required to be times of new generation of drug discovery and its analytical technologies [1].

\section{High-Throughput Screening (HTS): When it Becomes a Pitfall?}

The High-Throughput Screening (HTS) approach was regarded as the most powerful tool in processing the drug discovery. By identifying biologically active compounds against specific targets on an assay system with less complexity, the HTS approach offers pharmaceutical companies an in-house drug discovery strategy to select lead compound from a large scale of compound collections. Designed with the basis of "Key-Lock" principle, which was first described by Hermann Emil Fischer, the HTS approach could definitely develop a simple way to screen out leads against with a very high efficiency and productivity. Although the target-to-lead efforts in drug discovery, which involve the selection of targets, development of an assay, compound screening and validation of leads on a secondary assay, generally take 2 to 3 years in most of the pharmaceutical companies, the compound screening step using HTS approach typically takes from 1 week to 3 months. The great power of HTS allows it to finish a search amongst 1 million compounds within 3 months, and this power undoubtedly contributes to speed up the new drug development in pharmaceutical industries. Recent analysis found that 19 out of 58 approved drugs during 1991 to 2008 were contributed by HTS approach. However, the HTS approach has its own problems. Derived from the "Key-Lock" principle in nature, the HTS oversimplifies the complexity of the biological basis of a disease. The pathogenesis of a disease requires involvement of several pathways and a series of biological molecules, including hormones and receptors may contribute. Targeting at single target, therefore, may not make a treatment efficient. On the other hand, acquired mutation on a particular target may easily result in failure of drug treatment. The drug resistance effect is in consequence initiated. Өmitting the perturbation within the cellular network intentionally or not, the HTS may cause unanticipated off-target effect, leading to a serve adverse side effect during the treatment. These problems born in nature and seem to be hardly avoided during the development of HTS methodology, has restricted the application of HTS in pharmaceutical industry, when selective targets and ligands have been discovered in a great extent.
Increasing number of investigational new drugs fails in clinical trials because of the limited efficiency or serious adverse effects. Drug discovery and development with HTS approaches encounters a great challenge in the new era.

\section{Network Pharmacology: Choice for Pharmaceutical Industry in the New Era?}

The dilemma of HTS approach in drug discovery request a more comprehensive way of thinking to take into account with improved clinical efficacy and reduced side effect and toxicity. The idea of network pharmacology, as firstly described by Hopkins [2], embraces the concepts of polypharmacology and network biology together. The combination of polypharmacology, which was derived from the observation that most effective drugs act on multiple targets rather than single one, and network biology, which indicates modifying biological phenotype requires multiple modulation on a variety of cellular events, enables network pharmacology to be an approach for drug design that encompasses the systemic analysis on therapeutic targets, to avoid oversimplifying the biological events in a disease. The connectivity in nature allows this approach to oversee the cellular network perturbation.

For combination of multiple ingredients as new drugs, the challenge is multiple ingredients in the formulae [3]. Thus, it is hard to do research of multi-components drugs in bioequivalence and bioavailability. It is because bioequivalence and bioavailability studies can only compare the systemic situation of a test drug product with that of a reference drug product. Facing multi-components drugs, what can we do and how can we do bioequivalence and bioavailability studies? What new technologies we can apply to resolve such problems? Metabolomics is a powerful new technology that assesses holistic metabolic profiles in easily accessible biofluids and facilitates biomarker discovery to distinguish between diseased and non-diseased tissues. This will help to resolve the problem of multi-components drugs in bioavailability study. At the same time, other OMICS technologies will help-resolve problem of multiple components of Chinese medicines in bioequivalence study.

At technology level, network pharmacology employs a way with multiple layers in biological concepts, and embraces a variety of OMICS techniques, including genomics, transcriptomics, proteomics and metabolomics, to provide network topological parameter as foci and targets for hypothesis generation and assay design in drug discovery. With data mining technology, the possible targets of each component

*Corresponding author: Yibin Feng, LKS Faculty of Medicine, School of Chinese Medicine, The University of Hong Kong, 10 Sassoon Road, Pokfulam, Hong Kong, Tel: (852) 2589 0482; Fax: (852) 2872 5476; E-mail: yfeng@hku.hk

Received September 30, 2013; Accepted October 02, 2013; Published October 09, 2013

Citation: Feng Y, Wang N (2013) The New Generation of Drug Discovery and its Analytical Technologies. J Bioequiv Availab 5: e42. doi:10.4172/jbb.10000e42

Copyright: ( 2013 Feng Y, et al. This is an open-access article distributed under the terms of the Creative Commons Attribution License, which permits unrestricted use, distribution, and reproduction in any medium, provided the original author and source are credited. 
that are identified in pharmacokinetics could be included. The protein-protein interaction induced by multi-components drugs can be analyzed by bioinformatics software. This helps identify molecular basis of multi-component drugs in treating human diseases. Compared with the single layer approach of HTS, network pharmacology offers a scenario that mimics the actual biological process of the pathogenesis of a disease, which efforts to prove the clinical efficacy and adverse side effect of an investigational new drug. The application of network pharmacology approach in drug discovery may as a result increase the success rate of translating candidate leads into an approved drug.

\section{How can Traditional Medicine Contribute to the New Era of Drug Discovery?}

Traditional Medicine refers to knowledge systems of the use of medical materials that were developed beyond the era of modern medicine in a variety of societies with different cultures. Over generations, the knowledge of traditional medicine advanced and people in ancient societies, especially in Asian and African countries, require this kind of medicine for their primary health care needs. Traditional medicine incorporates plant, animal and mineral-based medication, physical and spiritual therapies and acupunctures, and a composite formula is the major form that traditional medicine practitioners applied to prevent and treat patient's diseases. Taken Traditional Chinese Medicine (TCM) as an example, a recent study summarized and analyzed the formula data in Dictionary of TCM, which includes 84183 prescriptions. Most of the prescriptions in TCM contain 4 to 9 components that are originated from medical herbs, parts of animals or minerals [4], and have been used for medical purpose for more than thousands of years with proved clinical efficacy and least adverse side effect and toxicity.

But the question remains how such an ancient science of health care could contribute to the modern therapy of human diseases? Treatment using traditional medicine is on the basis of the philosophy that the human body is a whole system and organs and tissues harmonize to gain a balance. This dynamic and connective view of traditional medicine gives a hint to link the ancient medical science to the modern drug discovery with network pharmacology approach. Considering their proved clinical efficacy and toxicity, the composite formulas from traditional medicine may test the validity of a network analysis. The process of validation could cycle iteratively for further data acquisition or network analysis. And a large pool of composite formulas with proved clinical efficacy and toxicity could also offer great candidates in drug screening, using network pharmacology approaches. Although the current methodologies may be effective in generating chemical candidates for singular treatment, candidates in appropriate combination, which have an extensive application in clinical practice, are hardly proved. The formulae from traditional medicine may therefore be definitely significant in find out candidates applied in combinations.

A good example has been provided in the study with RealgarIndigo naturalis formula (RIF) by Wang et al. [5]. The RIF is a Traditional Chinese Medicine formula that applied as an effective treatment against human acute promyelocytic leukemia (APL). The major active ingredients in RIF are tetraarsenic tetrasulfide (A), indirubin (I) and tanshinone IIA (T). Wang et al. [5] found that though $\mathrm{A}$ is the major active compounds in suppressing APL, I and T serve as adjuvant combination of ATI intensified ubiquitination/degradation of promyelocytic leukemia (PML)-retinoic acid receptor alpha (RARalpha) oncoprotein induced by $\mathrm{A}$, and $\mathrm{T}$ and $\mathrm{I}$, could additionally enhance the expression of Aquaglyceroporin 9 and facilitate the transportation of A. The work from Wang et al. [5] is deemed to be a milestone in the efforts of connecting ancient medicine with modern network pharmacology.

The quality issue of traditional medical formulae should be taken into consideration in applying them in the drug discovery with network pharmacology approach in this new era. A comprehensive and biological activity-relevant approach should be applied for the quality control of traditional medicine formulas. This method is definitely important in integrating the chemical response and bio-response of the formulae using network pharmacology approaches.

\section{Concluding Remarks}

The drug discovery process in pharmaceutical industry request to move towards a new era that integrates network pharmacology into the hypothesis generation and experiment design, since current tool of lead screening, the HTS approach, has shown some pitfalls. Traditional medicine shows a link with the network idea in drug discovery for its dynamic and connective philosophy in treatment. The application of traditional medicine into the modern drug discovery could not only cycle iteratively the validation on the network design of drug discovery, but also provide a series of candidates with proved clinical efficacy and toxicity. Multi-component drugs may display multiactions, which are studied by poly- pharmacology. New generation of drug discovery is required, new technologies in bioequivalence and bioavailability. Advanced analytical technologies have provided approaches in analyzing the bioactivities of single component drugs, but the complexity of chemical composition in multi-component drugs makes it difficult in identifying the chemical and biological basis underlying the pharmacological actions. Various measures have been developed and systems biology and OMICS are meaningful philosophy and technologies for this purpose. The quality issue should be taken into serious consideration, when applying traditional medicine in the modern drug discovery.

\section{Acknowledgement}

The study was supported by grants from the research council of the University of Hong Kong (Project Code: 10401764, 104002320), The Research Gran Committee (RGC) of Hong Kong SAR of China (RGC General Research Fund Project Code: 10500362), research donation from Wong's endowment and the Gaia Family Trust.

\section{References}

1. Feng $Y$ (2013) Advanced discussions of the current issues and analyzing the novel approaches of bioavailability and bioequivalence. J Bioequiv Availab 5: 33.

2. Hopkins AL (2007) Network pharmacology. Nat Biotechnol 25: 1110-1111.

3. Feng Y (2013) Bioavailability and bioequivalence studies for chinese medicines: Challenge and opportunity. J Bioequiv Availab 5: 54.

4. He P, Deng K, Liu Z, Liu D, Liu JS, et al. (2012) Discovering herbal functional groups of traditional Chinese medicine. Stat Med 31: 636-642.

5. Wang L, Zhou GB, Liu P, Song JH, Liang Y, et al. (2008) Dissection of mechanisms of Chinese medicinal formula Realgar-Indigo naturalis as an effective treatment for promyelocytic leukemia. Proc Natl Acad Sci USA 105: 4826-4831. 